\title{
DOS ESPECIES NUEVAS DE LOBELIA (CAMPANULACEAE) DE MEXICO'
}

\author{
Jerzy Rzedowski y Graciela Calderon de Rzedowski \\ Instituto de Ecología, A.C. \\ Centro Regional del Bajío \\ Apartado postal 386 \\ 61600 Pátzcuaro, Michoacán
}

\begin{abstract}
RESUMEN
Se describen como nuevas e ilustran Lobelia diazlunae, de la Sierra Madre Occidental, y $L$. orientalis, de la Sierra Madre Oriental, ambas solamente conocidas de la localidad tipo, representando con toda probabilidad muy estrechos endemismos. La primera, propia del NE de Nayarit, tiene semejanza con L. nana H.B.K. así como con L. standleyi McVaugh, en función de su hábito rastrero y flores solitarias. La segunda se ha descubierto en el NE de Querétaro y se relaciona con L. tarsophora Seaton, así como con L. hypnodes Wimmer, en vista de su ovario y cápsula súperos y de sus semillas foveolado-reticuladas.
\end{abstract}

\section{ABSTRACT}

Lobelia diazlunae, from the Sierra Madre Occidental, and L. orientalis, from the Sierra Madre Oriental of Mexico, are described as new and illustrated. Most probably both represent very narrow endemics. The first one, collected in northeastern Nayarit, on account of its prostrate habit and solitary flowers, is similar to L.nana H.B.K. and to L. standleyi McVaugh. The second, discovered in northeastern Querétaro, in view of its superior ovary and capsule, as well of its foveolate-reticulate seeds, is related to $L$. tarsophora Seaton and L. hypnodes Wimmer.

La preparación del manuscrito de la familia Campanulaceae para la Flora del Bajío y de regiones adyacentes y la consiguiente revisión de amplios materiales de este grupo en los herbarios mexicanos, permitieron encontrar representantes de varias especies del género Lobelia, que no figuran en las obras monográficas de McVaugh (1942) y de Wimmer (1953). Dos de estas novedades se describen a continuación.

Lobelia diazlunae Rzedowski \& Calderón sp. n. (Fig. 1)

Herba perennis rhizomatosa prostrata; caules plures ad $20 \mathrm{~cm}$ longi hirsutuli; folia breviter petiolata plerumque suborbicularia (2)2.5-4(5.5) $\mathrm{cm}$ longa et lata grosse crenatodentata vel subintegra glabra; flores solitarii axillares, pedunculi 1-3 cm longi ebracteolati;

${ }_{1}^{1}$ Trabajo realizado con apoyo económico del Instituto de Ecología, A.C. (cuenta 902-03), del Consejo Nacional de Ciencia y Tecnología y de la Comisión Nacional para el Conocimiento y Uso de la Biodiversidad. 
hypanthium in anthesi turbinatum, calycis lobi oblongi 3-3.5 $\mathrm{mm}$ longi integri; corolla e purpureo albida, tubo 3-4 mm longo non fenestrato, lobulis 2-2.5 mm longis; filamentorum tubus ca. $3 \mathrm{~mm}$ longus, antherae ca. $1.5 \mathrm{~mm}$ longae sparsim puberulae, 2 inferiores apice barbatae; capsula subfusiformis 5-6 mm longa; semina ellipsoidea ca. $0.4 \mathrm{~mm}$ longa brunnea laevia nitida.

Planta herbácea perenne, rastrera, provista de largos rizomas horizontales delgados, de los que parten finas raicillas tortuosas; tallos numerosos, extendiéndose en forma radial, hasta de $20 \mathrm{~cm}$ de largo, de poco menos de $1 \mathrm{~mm}$ de diámetro, mayormente de color pajizo, provistos de pelos rígidos, finos, de 0.1 a $0.2 \mathrm{~mm}$ de largo; peciolos de 1 a $2 \mathrm{~mm}$ de largo, láminas foliares mayormente orbiculares, variando de anchamente deltoideo-ovadas a reniformes, de (2)2.5 a 4(5.5) mm de largo y otro tanto de ancho, ápice obtuso a redondeado, a veces casi truncado y apiculado, base por lo general truncada, pero variando de cuneada a subcordada, margen toscamente crenado-dentado a subentero, venación palmada, textura membranácea, glabras; flores solitarias en las axilas de las hojas, pedúnculos de 1 a $3 \mathrm{~cm}$ de largo, con pubescencia similar a la de los tallos, bracteolas ausentes; hipantio turbinado en la antesis, de poco más de $1 \mathrm{~mm}$ de largo y de ancho, lóbulos del cáliz oblongos, de 3 a $3.5 \mathrm{~mm}$ de largo, agudos en el ápice, enteros en el margen, glabros; corola blanquecina con tintes morados hacia la parte distal, glabra, tubo de 3 a $4 \mathrm{~mm}$ de largo, hendido dorsalmente casi hasta la base, no fenestrado, lóbulos superiores lanceolados, de 2 a $2.5 \mathrm{~mm}$ de largo, lóbulos inferiores angostamente ovados, de aproximadamente la misma longitud; tubo de los filamentos de unos $3 \mathrm{~mm}$ de largo, pálido, sin perder su integridad en la base, anteras de aproximadamente $1.5 \mathrm{~mm}$ de largo, moradas, esparcidamente pubérulas, las más cortas barbadas en el ápice; cápsula subfusiforme, de 5 a $6 \mathrm{~mm}$ de largo, ínfera en sus 2/3 partes; semillas elipsoides, levemente comprimidas, de aproximadamente $0.4 \mathrm{~mm}$ de largo, de color café, lisas, brillantes.

TIPO: México. Nayarit. Santa Anita, $11 / 2 \mathrm{~km}$ al NE de Santa Teresa del Nayar, municipio de Jesús María, terreno pedregoso, alt. 1800 m, 11.VI.1978, C. L. Díaz Luna 9572 (holotipo ENCB, isotipo GUADA).

En su porte rastrero y en sus flores solitarias en las axilas de las hojas, L. diazlunae se asemeja a L. nana H.B.K., que se distribuye de México a Sudamérica, y a L. standleyi McVaugh, conocida de Guatemala, pero difiere de ambas en la forma de las hojas y en los tallos no radicantes. Esta similitud no necesariamente refleja verdaderos vínculos de parentesco.

De acuerdo con Wimmer (1953, pp. 486-487), en el Antiguo Mundo existen dos especies de Lobelia con hábito y hojas similares, a mencionar: L. minutula Engl., conocida de Africa, y L. brachyantha Merrill \& Perry, descrita de Nueva Guinea. Ambas difieren de la que aquí se describe en sus flores más pequeñas y moradas, así como en sus pedúnculos mucho más cortos.

L. diazlunae sólo se registra de una colecta realizada en la remota región cora-huichol del NE de Nayarit, cercana a los límites de Durango y de Jalisco. Probablemente se trata de un estrecho endemismo de la Sierra Madre Occidental. 
Rzedowski y Calderón de Rzedowski: Dos Especies Nuevas de Lobelia de México
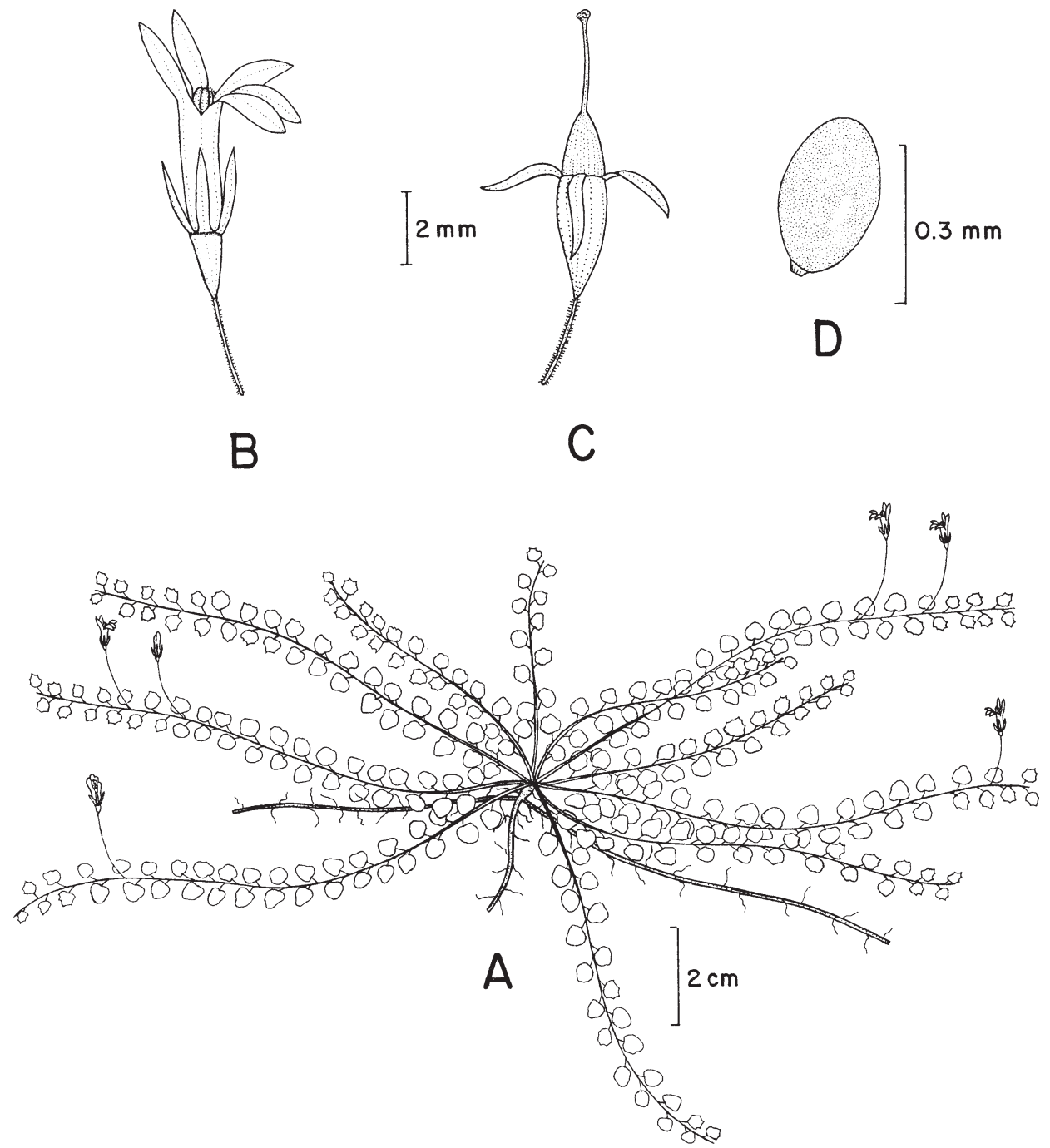

Fig 1. Lobelia diazlunae Rzedowski \& Calderón. A. rama con flores; B. flor; C. fruto; D. semilla. llustración de Rogelio Cárdenas.

El nombre de la especie está dedicado a la memoria del recientemente desaparecido Biól. Carlos Luis Díaz Luna, durante muchos años catedrático de la Universidad Autónoma de Guadalajara y colector de esta interesante planta. El Biól. Díaz Luna se destacó por su labor docente y en el campo de la botánica e incursionó con éxito en la taxonomía de la familia Caricaceae. 
Lobelia orientalis Rzedowski \& Calderón sp. n. (Fig. 2)

Herba perennis subscaposa gregaria rhizomatosa hirsutula ad 10(20) cm alta; caules plures, internodis abbreviatis celeriter in inflorescentias transformantes; folia ad plantae basem convergentia longe petiolata, laminis plerumque ovatis (1.2)1.5-2(3.5) cm longis, (0.8)1-1.5(2.2) cm latis margine subintegris usque ad denticulatis utrinque pubescentibus; racemi laxi non secundiflori, pedicellis (1)1.5-2.5(3.5) longis, ebracteolatis; hypanthium subnullum, calycis lobi anguste triangulares 1-2(3) mm longi glabri; corolla e purpureo alba, tubo ca. $5 \mathrm{~mm}$ longo non fenestrato, lobis inferioribus obovatis $2-4 \mathrm{~mm}$ longis, lobis superioribus pauce brevioribus oblongis; antherae 1.5-2 $\mathrm{mm}$ longae puberulae, 2 inferiores apice barbatae; ovarium superum; capsula late ovoidea $4-5 \mathrm{~mm}$ longa omnino supera; semina breviter ellipsoidea ca. $0.5 \mathrm{~mm}$ longa pallide brunnea minute foveolato-reticulata nitida.

Planta herbácea perenne, subescaposa, hasta de 10(20) cm de alto, creciendo varios individuos muy juntos, con frecuencia unidos por medio de rizomas, provista de pubescencia hirsútula blanquecina de pelos simples, multicelulares, en tallos, hojas y pedúnculos, pero especialmente densa en la porción basal de la planta; raíz pivotante inicialmente evidente, pero con la edad, un amplio conjunto de raíces fasciculadas parte de una base rizomatosa; tallos por lo general saliendo varios desde la base, con los entrenudos muy cortos y pronto convirtiéndose en ejes de las inflorescencias; hojas alternas, todas agrupadas en la base de la planta, peciolos delgados, hasta de $4(4.5) \mathrm{cm}$ de largo, láminas foliares mayormente ovadas, variando de ampliamente oblongas a suborbiculares, de (1.2)1.5 a 2(3.5) cm de largo y (0.8)1 a 1.5(2.2) cm de ancho, ápice agudo a obtuso, raras veces redondeado o emarginado, con frecuencia provisto en la punta de un callo a modo de mucrón diminuto, base redondeada a truncada, a menudo abrupta y cortamente decurrente, margen subentero a ondulado o denticulado-calloso, de textura membranácea, con pubescencia en ambas caras, en el envés principalmente a lo largo de las nervaduras; flores dispuestas en racimos laxos, no secundifloros, provistos de brácteas subuladas a lineares de 1 a 2(3) mm de largo, pedicelos de (1)1.5 a $2.5(3.5) \mathrm{cm}$ de largo, bracteolas ausentes; cáliz ligeramente unido en la base, hipantio obsoleto, lóbulos subiguales, linear-subulados a estrechamente triangulares, de 1 a 2(3) $\mathrm{mm}$ de largo, enteros en el margen, glabros; corola blanca con tintes violáceos o morados hacia la parte distal, glabra, tubo de alrededor de $5 \mathrm{~mm}$ de largo, hendido dorsalmente hasta cerca de la base, no fenestrado, lóbulos inferiores obovados, de (2)3 a $4 \mathrm{~mm}$ de largo, los superiores angostamente oblongos, por lo general de igual largo o un poco más cortos; tubo de los filamentos blanquecino, de 4 a $5 \mathrm{~mm}$ de largo, sin perder su integridad en la base (en la antesis), anteras moradas, de 1.5 a $2 \mathrm{~mm}$ de largo, pubérulas, las más cortas barbadas en el ápice; ovario súpero; cápsula totalmente súpera, ampliamente ovoide, de 4 a $5 \mathrm{~mm}$ de largo, algo truncada en la base, apiculada en la punta; semillas cortamente elipsoides, de aproximadamente $0.5 \mathrm{~mm}$ de largo, de color café pálido, diminutamente foveolado-reticuladas, brillantes.

TIPO: México. Querétaro. Llano Chiquito, municipio de Landa, bosque mesófilo de montaña sobre laderas de rocas calizas kársticas, $21^{\circ} 23^{\prime} \mathrm{N}, 99^{\circ} 06 \mathrm{~W}$, alt. $2000 \mathrm{~m}$, 1-2.IV.1997, S. Zamudio y E. Carranza 10200 (holotipo IEB, isotipos por distribuirse). 


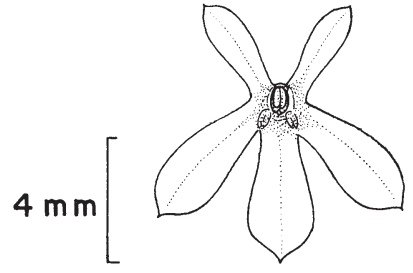

C

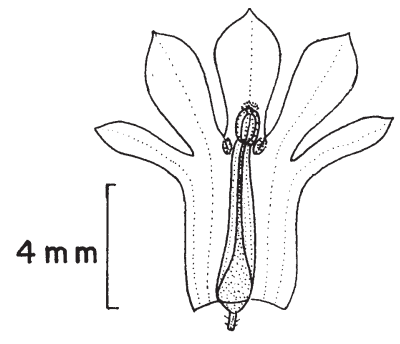

D

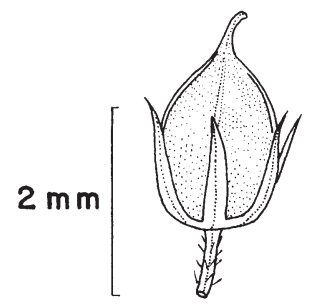

$E$

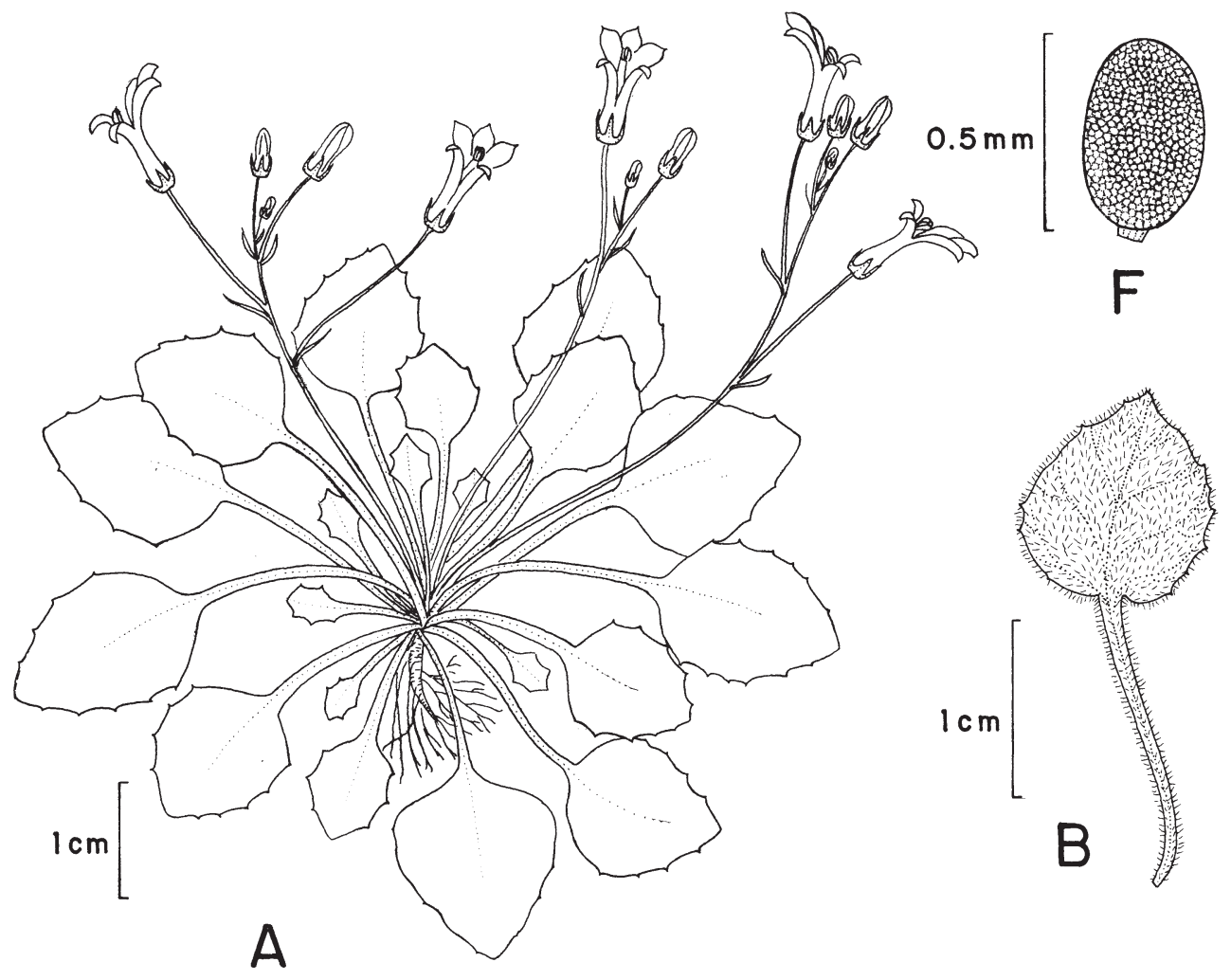

Fig. 2. Lobelia orientalis Rzedowski \& Calderón. A. planta entera; B. hoja; C. limbo de la corola; D. corola disecada, mostrando el androceo y el gineceo; E. cápsula; F. semilla. Ilustración de Rogelio Cárdenas. 
Material adicional examinado: México. Querétaro. Llano Chiquito, municipio de Landa, bosque de Cupressus y Pinus sp., sobre laderas calizas kársticas, alt. $1980 \mathrm{~m}$, 17.II.1989, S. Zamudio y E. Carranza 7142 (IEB).

La especie sólo se conoce de la localidad tipo, ubicada en el extremo NE del estado de Querétaro sobre el límite con San Luis Potosí. Se ha registrado como localmente abundante, creciendo entre grietas de rocas calizas, en medio del bosque de coníferas y del bosque mesófilo de montaña. Muy verosímilmente representa un estrecho endemismo de la Sierra Madre Oriental.

Lobelia orientalis descuella por su ovario y cápsula totalmente súperos, en combinación con semillas foveolado-reticuladas y porte subescaposo. El primero de los mencionados caracteres se presenta rara vez en el género y en la familia entera. Por otro lado, la presencia de semillas ornamentadas no permite ubicar de manera cómoda a la especie en la sección Hemipogon, a la que pertenecen prácticamente todos los demás miembros mexicanos de Lobelia con flores azules, moradas o blancas. Sin embargo, como ya lo señaló McVaugh (1942, pp. 64-65), L. tarsophora Seaton y L. hypnodes Wimmer presentan este mismo carácter y por consiguiente con toda probabilidad están emparentadas con L. orientalis. L. hypnodes, conocida solamente del norte de Veracruz, también destaca por su ovario y cápsula súperos, mientras que L. tarsophora, distribuida a lo largo de la Sierra Madre Oriental de San Luis Potosí a Hidalgo, Puebla y Veracruz, conserva un hipantio relativamente desarrollado al menos en fruto, que es ínfero en su cuarta o tercera parte. L. orientalis difiere de ambas especies de manera notable en ser planta perenne de porte pequeño, subescaposo y gregario.

Cabe comentar, al margen de lo anterior, que la tendencia hacia la reducción del hipantio y la consiguiente ubicación súpera o casi súpera del ovario se ha manifestado aparentemente en varias líneas evolutivas de la sección Hemipogon de Lobelia. El fenómeno puede notarse en L. diastateoides McVaugh, L. homophylla Wimmer, L. pulchella Vatke y L. dielsiana Wimmer, además de L. poetica Wimmer, pero estas especies no están cercanamente emparentadas con el grupo de L. hypnodes, L. tarsophora y L. orientalis.

\section{AGRADECIMIENTOS}

Se agradece a los responsables de los herbarios ENCB y GUADA el préstamo de sus materiales de Lobelia, importantes para la preparación de este trabajo. Los Bióls. Sergio Zamudio y Eleazar Carranza realizaron un viaje especial con el propósito de localizar muestras con flor y fruto de L. orientalis. El Dr. Rogers McVaugh tuvo la amabilidad de examinar los ejemplares de ambas especies y de opinar sobre su carácter distintivo.

\section{LITERATURA CITADA}

McVaugh, R. 1942. Campanulaceae (Lobelioideae). North Amer. FI. 32A: 1-134.

Wimmer, F. E. 1953. Campanulaceae - Lobelioideae. Pflanzenr. 107. IV. 276b: 261-813. 